\section{Fragilidad: en busca de herramientas de evaluación preoperatoria}

\author{
JAVIERA VARGAS ${ }^{1,2}$, MARÍA DE LOS ÁNGELES GÁLVEZ ${ }^{1, a}$, \\ MARIANA ROJAS ${ }^{1, a}$, MACARENA HONORATO ${ }^{3}$, \\ MARICARMEN ANDRADE ${ }^{4}$, PATRICIO LEYTON ${ }^{1}$, \\ GABRIELA MARDONES ${ }^{2, a}$, JULIÁN MORALES ${ }^{2, a}$, \\ DANIELA PÉRSICO ${ }^{2, a}$, FERNANDA ROJAS ${ }^{2}$, DUBY MORENO², \\ ERIKA BECKER ${ }^{2}$, GABRIEL CAVADA $^{\mathrm{b}}$, CRISTÓBAL CARVAJAL
}

\section{Assessment of two frailty scales for the preoperative period}

Background: In the perioperative context, a frailty evaluation scale must consider certain characteristics such as validation, execution speed, simplicity, the capacity to measure multiple dimensions and not being dependent on a cognitive or physical test that could not be performed prior to surgery. The test should select patients that could benefit from interventions aimed to improve their postoperative outcomes. Aim: To validate two frailty evaluation scales for the perioperative period. Material and Methods: The Risk Analysis Index with local modifications (RAI-M) were applied to 201 patients aged $73 \pm 7$ years (49\% women) and the Edmonton frailty scale were applied in 151 patients aged $73 \pm 7$ years (49\% women) in the preoperative period. Their results were compared with the Rockwood frailty index. Results: The Edmonton frail scale showed adequate psychometric properties and assessed multiple dimensions through 8 of the 11 original questions, achieving a discrimination power over $80 \%$ compared to the Rockwood Index. The RAI- M, demonstrated solid psychometric properties with a tool that examines 4 dimensions of frailty through 15 questions and reviewing the presence of 11 medical comorbidities. This scale had a discrimination power greater than $85 \%$ and it was significantly associated with prolongation of the planned hospital stay and mortality. Conclusions: RAI-M is a short and easily administered scale, useful to detect frailty in the preoperative period.

(Rev Med Chile 2020; 148: 311-319)

Key words: Anesthesia; Frail Elderly; Perioperative Care; Perioperative Period; Risk Assessment.
'Servicio de Anestesiología. Universidad del Desarrollo-Clínica Alemana Santiago. Santiago, Chile.

${ }^{2}$ Servicio de Anestesiología. Universidad Diego PortalesHospital Barros Luco Trudeau.

Santiago, Chile.

${ }^{3}$ Unidad de Geriatría.

Departamento de Medicina

Interna. Universidad del

Desarrollo-Clínica Alemana

Santiago. Santiago, Chile.

${ }^{4}$ Unidad de Geriatría.

Departamento de Medicina

Interna. Clínica Universidad de los

Andes. Santiago, Chile.

${ }^{a}$ Residente Anestesiología.

${ }^{b}$ Magíster en Bioestadística.

Escuela Salud Pública.

Universidad de Chile.

'Magíster en informática médica.

Con apoyo financiero de la Universidad del Desarrollo, quien no tuvo influencia en el diseño del estudio, ni en la recolección de datos, ni en el análisis de éstos, así como tampoco en la preparación del manuscrito.

Recibido el 3 de septiembre de 2019, aceptado el 9 de marzo de 2020.

Correspondencia a: Javiera Paola Vargas Zúñiga Av. Vitacura 5951. Vitacura. Santiago, Chile. jpvargasz@gmail.com egún las estimaciones del INE, para el 2050 la población mayor de 65 años se duplicará respecto al 2018, llegando a corresponder a $25 \%$ de la población ${ }^{1}$. El equipo médico se verá enfrentado, con mayor frecuencia, a otorgar cuidados perioperatorios en pacientes con condiciones particulares que puedan afectar sus resultados postquirúrgicos.

Dentro de este grupo etáreo, el diagnóstico de fragilidad ha tomado relevancia, ya que este estado de menor reserva fisiológica y menor capacidad de respuesta al estrés, quirúrgico en nuestro contexto, se asocia con peores resultados postoperatorios, con un aumento en la morbimortalidad ${ }^{2,3}$. Parece importante poder pesquisar tempranamente a estos pacientes y ofrecer un manejo integral multidisciplinario durante el perioperatorio para mejorar los resultados. 
El estándar de oro en el diagnóstico de fragilidad es una evaluación geriátrica integral, que requiere tiempo y especialistas que no siempre están disponibles. Existen múltiples escalas para medición de fragilidad mundialmente conocidas: el fenotipo clínico de fragilidad desarrollada por Linda Fried ${ }^{2}$, evalúa sólo el dominio físico requiriendo medición de fuerza prensil y velocidad de marcha; el índice de fragilidad (IF) de Rockwood ${ }^{4}$ es multidimensional, pero consta de una valoración geriátrica integral; y la escala Edmonton Frail Scale $(\mathrm{EFS})^{5-7}$ validada en el preoperatorio, considera la prueba "time up and go" (TUG). Todas las anteriores requieren de tiempo y condiciones que una proporción considerable de las evaluaciones preoperatorias no tienen.

Según Rockwood y su IF basado en la acumulación de déficit, un puntaje de corte de 0,3 o más demuestra asociación con fragilidad, considerando su valor como una proporción de déficit respecto al total de ítems evaluados ${ }^{8}$; originalmente descrito con el análisis de 92 ítems ${ }^{9}$, actualmente validado su uso con un mínimo de 30 ítems ${ }^{10}$.

La sarcopenia es otra característica habitual en pacientes frágiles. Hay distintas formas de evaluarla a través de asociaciones con pruebas únicas: fuerza prensil medida con dinamometría ${ }^{11}$, incorporada en el fenotipo clínico de fragilidad, validada en población chilena $(<15 \mathrm{~kg}$ en mujeres, $<27 \mathrm{~kg}$ en hombres) ${ }^{12}$; circunferencia de pantorrilla, estandarizada en población sudamericana con un punto de corte de $<31 \mathrm{~cm}^{13,14}$; y el TUG con un punto de corte mayor a 20 segundos ${ }^{15}$.

Una herramienta para evaluar fragilidad en el perioperatorio debe tener las siguientes características: validez, rapidez de ejecución, sencillez, capacidad de medir múltiples dimensiones y no depender de una prueba cognitiva o física que puede no ser factible previo a una cirugía. En este contexto destaca la escala Risk Analysis Index (RAI), una herramienta que posee las características deseables mencionadas. Fue desarrollada a partir de otra escala, la Minimum data set mortality index (MMRI) ${ }^{3}$, que se validó para predecir mortalidad en pacientes que ingresaban a instituciones de larga estadía. Posteriormente la escala RAI fue modificada y validada por otros investigadores observando asociación con mortalidad y morbilidad postoperatoria en pacientes frágiles ${ }^{16-18}$.

Nuestro objetivo es detectar fragilidad en AM de forma preoperatoria aproximándonos a la valoración geriátrica integral, con una herramienta fácil de realizar, para poder identificar dominios que evidencien al paciente vulnerable al estrés quirúrgico.

En el presente estudio evaluaremos en población chilena de un centro, el comportamiento de la EFS y la escala RAI-M (adaptada desde la escala RAI) en comparación al IF de Rockwood considerado como el estándar. Ambas escalas están compuestas de preguntas dicotómicas, sencillas y rápidas que interrogan distintas dimensiones para identificar el paciente frágil.

\section{Material y Métodos}

Se realizó la traducción y transculturación al español de la escala RAI por dos profesionales bilingües. Posteriormente se evaluó su validez de contenido mediante el criterio de jueces expertos con el fin de evaluar la claridad, relevancia y pertinencia de los ítems. Sobre ésta, se elaboró la escala RAI-M con un equipo multidisciplinario, adicionando preguntas que buscaban ampliar la evaluación de fragilidad desde su perspectiva multifactorial definiendo las dimensiones: Física, nutricional, social, afectiva, cognitiva y funcional, además de evaluar la carga de patología médica. Este instrumento posee 11 preguntas de antecedentes médicos, 25 de respuesta dicotómica y no requiere de pruebas físicas o cognitivas (Anexo 1).

Aprobado por Comité de Ética de la Investigación del Centro de Bioética de la Facultad de Medicina de Clínica Alemana - Universidad del Desarrollo se procedió a la prueba experimental. Se incluyeron AM de 65 años o más programados para un procedimiento electivo con anestesia, que acepte participar y que haya estado en condiciones de realizar la evaluación o hubiese un familiar o cuidador a cargo que pueda responder por él. Se excluyeron los pacientes hospitalizados para cirugía de emergencia, que se negaron a participar o que no estuvieron en condición de realizar la evaluación. Las escalas fueron realizadas por médicos, kinesiólogos y fonoaudiólogos.

Paralelamente se realizó la traducción, valoración por jueces expertos, muestra piloto, corrección y posterior evaluación en muestra experimental de EFS (Anexo 2).

Como estándar de oro se realizó un IF de Roc- 
kwood de 31 ítems (Anexo 3), incluyó preguntas del área cognitiva (test del reloj), nutricional, funcional, social y antecedentes médicos. Además, se midió Índice de Masa Corporal, circunferencia de pantorrilla, dinamometría y TUG, pruebas objetivas utilizadas como herramienta única para medir sarcopenia en estudios previos ${ }^{10}$ (Anexo 4).

Las respuestas obtenidas fueron almacenadas en formato digital (Redcap), a la cual solo tenía acceso el investigador principal para protección de datos.

\section{Tamaño muestral}

El tamaño de muestra experimental, según la recomendación más habitual, se empleó una muestra 10 veces mayor al número de variables $(\mathrm{N}=10 \mathrm{k}$, donde $\mathrm{k}$ es el número de ítems o variables) ${ }^{19,20}$. Sin embargo, otros autores creen suficiente una muestra menor ${ }^{21,22}$; dos o tres veces el número de ítems o variables $(\mathrm{N}=2 \mathrm{k}$ o $\mathrm{N}=3 \mathrm{k})$, con la condición de que el número de sujetos no sea inferior a 200.

Se estimó un tamaño muestral experimental mínimo de 200 para RAI-M y 110 para EFS.

\section{Análisis estadístico}

Para caracterizar la muestra se obtuvieron medias y DS o medianas y percentiles según la distribución de los datos y para las variables categóricas se obtuvo frecuencias absolutas y relativas.

El puntaje de RAI-M se obtiene contando la cantidad de ítems positivos de las distintas dimensiones evaluadas, varía entre 0-25 puntos. El puntaje EFS se obtiene sumando las respuestas individuales de 11 ítems valorados de $0-2$, este puntaje va de 0-17 puntos.

Se buscó evidencia de validez de RAI-M y EFS al evaluar su constructo, su capacidad de discriminación y predictiva.

Se evaluó la consistencia interna mediante alfa de Cronbach para todos los ítems, se realizó Análisis Factorial Exploratorio y confirmatorio para la validación de constructo según teoría clásica reportando índices de bondad de ajuste.

Para buscar evidencia de validez por convergencia se evaluó la capacidad de discriminación de EFS y RAI-M respecto de la condición de fragilidad como respuesta dicotómica de la escala de Rockwood con un IF de 0.3 mediante el área bajo la curva (AUC) ROC.

Se obtuvieron los días de hospitalización, rehospitalización, prolongación de hospitalización para evaluar la validez predictiva de estas herramientas a través de modelos de regresión logístico, múltiples y la capacidad de discriminación mediante el análisis del área bajo la curva (AUC) ROC.

Se evaluó el tiempo al evento de muerte a un seguimiento máximo de 9 meses con variable pronóstico el score RAI-M ajustado y luego dicotomizado según punto de discriminación con el Gold standard, mediante un modelo de riesgos proporcionales de Cox. También se evaluó el tiempo al evento de muerte a un seguimiento máximo de 9 meses con EFS como variable pronóstico en un modelo independiente.

Se utilizó una significación de 5\%, intervalos de confianza de nivel 95\%. Los datos fueron procesados en STATA 14.0.

\section{Resultados}

\section{RAI-M}

Muestra experimental de 201 pacientes, edad promedio fue de $73+/-7$ años, distribución de $51 \%$ hombres y $49 \%$ mujeres, el porcentaje de pacientes clasificados como frágiles según Rockwood es de un 35\%. Las patologías reportadas por los pacientes se observan en la Tabla 1.

Tabla 1. Grupo de validación RAI-M

\begin{tabular}{|ll|}
\hline Total de pacientes & 201 \\
\hline Edad & $73 \pm 7$ años \\
\hline Distribución por sexo & $51 \%$ hombre y 49\% mujeres \\
\hline Fragilidad por Rockwood & $35 \%$ \\
\hline Comorbilidades & HTA: $54 \%$ \\
& DM: $19 \%$ \\
& Cáncer: $39 \%$ \\
& EPOC: $5 \%$ \\
& IAM: 3,5\% \\
& Angina: $2,5 \%$ \\
Insuficiencia cardíaca: $6,6 \%$ \\
Asma: $2,5 \%$ \\
Artritis: $13,5 \%$ \\
ACV: $4,5 \%$ \\
ERC: 3,5\% \\
\hline
\end{tabular}


$\mathrm{Al}$ evaluar confiabilidad interna observamos los siguientes valores: 0,92 para dimensión físico-nutricional, 0,78 afectivo, 0,72 funcional $y$ 0,64 social.

Se evaluó la estructura de la escala RAI-M donde el análisis factorial exploratorio arroja una estructura preliminar de 4 factores. Tras eliminar los ítems con pesos factoriales menores a 0,3 y los ítems que presentaban pesos factoriales significativos en más de una dimensión se obtuvo una estructura definida por 4 dimensiones: Físico-nutricional, afectiva, social y funcional con un total de 15 preguntas de las 25 iniciales. El análisis factorial confirmatorio arroja adecuados índices de bondad de ajuste: RMSEA $=0,028, T L I=0,90 \mathrm{y}$ $\mathrm{CFT}=0,92$, que se traduce en que los indicadores miden en conjunto cada una de estas dimensiones, $y$ en total, el riesgo de fragilidad como fue definido teóricamente en la creación de la escala con algunas modificaciones, tales como la exclusión de la dimensión cognitiva y de algunas preguntas que interrogan aspectos físicos principalmente.

$\mathrm{Al}$ evaluar la escala RAI-M buscando validez por convergencia en relación al IF, se observó una asociación significativa en un modelo logístico con un OR de 1,88 (IC: 1,28-2,74) y un poder de discriminación de $87 \%$ (IC: 0,81-0,92) con una sensibilidad de $78 \%$ y una especificidad de $85 \%$ para un punto de corte de 3 .

En cuanto a la validez predictiva se observó asociación con prolongación de hospitalización, con un OR ajustado por patologías médicas y edad de 1,45 (IC: 1,01-1,88). No se asoció con rehospitalización.

Al evaluar el score como pronóstico de muerte a 9 meses, ajustado por edad, género, riesgo quirúrgico y carga de patología médica se encontró que solo el score RAI-M era estadísticamente significativo, $\mathrm{HR}=1,58$ ( $\mathrm{p}=0,013$ IC: $1,10-2,27)$; este riesgo de morir explicado a través del score RAI-M dicotomizado en 3 o más puntos fue de $\mathrm{HR}=4,98(\mathrm{p}=0,029$ IC: $1,17-2,13)$.

\section{EFS}

Muestra experimental de 151 pacientes, edad promedio fue de $73+/-7$ años, distribución de $51 \%$ hombres y $49 \%$ mujeres, el porcentaje de pacientes clasificados como frágiles según Rockwood es de un $41 \%$. Patologías reportadas por los pacientes se observan en la Tabla 2.

En el análisis de su constructo se observó una estructura de un factor con buenos índices de bondad de ajuste RMSEA $=0,01, \mathrm{CFI}=0,99 \mathrm{y}$ $\mathrm{TLI}=0,99$. Se eliminaron los ítem $5\left({ }_{i}\right.$ Cuando usted necesita ayuda, puede contar con alguien que esté dispuesto y disponible para atender sus necesidades o problemas?), ítem 7 (En ocasiones, ¿se le olvida tomarse los medicamentos?) y el ítem 10 (¿Tiene algún problema con el control para orinar, es decir, puede contener la orina si así lo desea?) por tener bajo peso factorial menor de 0,3 . $\mathrm{Al}$ evaluar el modelo sin retirar los 3 ítems mencionados para mantener la estructura original se conservan los buenos índices de bondad de ajuste.

La concordancia interna es de 0,66 para EFS con 8 ítems (y de 0,64 con 11 ítems originales).

Como evidencia de validez por convergencia para el EFS con 8 ítems se observó una asociación significativa en un modelo logístico con un OR de 2,3 (IC 95\%: 1,7-3,0), con un poder de discriminación de un 88\% (IC 95\%: 0,82-0,93), y con una sensibilidad de $79 \%$ y especificidad de $81 \%$ para un punto de corte de 5 puntos o más.

En cuanto a la validez predictiva no se observó asociación con prolongación de hospitalización o con rehospitalización. Al evaluar el score EFS como variable pronóstico de muerte a 9 meses a través de un modelo de riesgos proporcionales de Cox se observó que EFS es estadísticamente significativa con un $\mathrm{HR}=1,56(\mathrm{p}=0,004$ IC: $1,15-2,12)$.

Tabla 2. Grupo de validación EFS

\begin{tabular}{|ll|}
\hline Total de pacientes & 151 \\
\hline Edad & $73 \pm 7$ años \\
\hline Distribución por sexo & $51 \%$ hombre y 49\% mujeres \\
\hline Fragilidad por Rockwood & $41 \%$ \\
Comorbilidades & HTA: $54 \%$ \\
& DM: $18 \%$ \\
& Cáncer: $38 \%$ \\
& EPOC: $5,9 \%$ \\
& IAM: $2,6 \%$ \\
& Angina: $1,3 \%$ \\
Insuficiencia cardíaca: 6,6\% & Asma: 3,3\% \\
Artritis: $15,2 \%$ \\
ACV: $4,6 \%$ \\
ERC: 3,3\% \\
\hline
\end{tabular}




\section{Discusión}

La fragilidad ha demostrado ser un importante factor predictor de desenlaces adversos en el ámbito del paciente quirúrgico. Se evaluaron distintas herramientas para determinar fragilidad en el contexto periquirúrgico, en busca de un instrumento que midiera las dimensiones de fragilidad en forma rápida, sencilla y sin pruebas físicas o cognitivas, tratando de acercarnos a la valoración geriátrica integral.

RAI-M muestra concordancia interna adecuada en las distintas dimensiones a evaluar; tiene evidencia de validez de constructo con la modificación a 4 dimensiones; validez por convergencia con el IF de Rockwood y con EFS. Muestra una asociación predictiva con desenlaces de interés como mortalidad y prolongación de la hospitalización.

EFS, cumple con las características deseables de una herramienta de evaluación en el contexto peri-quirúrgico, excepto por la necesidad de realizar pruebas físicas y cognitivas, obtuvo buenos resultados en su validez de constructo, pero 3 ítems se descartan en el análisis clásico. Muestra evidencia de validez por convergencia con el estándar de oro.

Las preguntas que evaluaron cognición fueron eliminadas porque no aportaba en la escala de fragilidad, esto nos llamó la atención por la correlación ya conocida entre fragilidad y deterioro cognitivo $^{21}$, pensamos que puede deberse a que los pacientes no reconocen sus déficits cognitivos en etapas iniciales, por lo que la pregunta hecha al paciente tiene poca validez, en nuestro análisis secundario, hubo mejor correlación de las respuestas que evalúan este dominio cuando se hacía la pregunta al familiar/cuidador. Se evidenció que las fallas más complejas de las actividades instrumentales (apoyo en manejo de fármacos y dinero), son poco sensibles para detectar fragilidad, a diferencia de la afectación en las actividades básicas de la vida diaria. La única actividad básica que no se asoció a fragilidad fue la incontinencia urinaria, probablemente porque su frecuencia en AM es aproximadamente un $30 \%$, principalmente mujeres y no reflejan necesariamente vulnerabilidad a menos que aparezca tardíamente en contexto de alguna enfermedad neurodegenerativa o por alteración funcional.

La fragilidad es un continuo entre el paciente robusto y el frágil, es probable que con las herramientas que estamos utilizando actualmente para medirla, así como los desenlaces que estamos evaluando, como son muerte y hospitalización, no logren detectar cambios menores en este continuo. Esto no significa que alteraciones de memoria y de funcionalidad no se asocien a fragilidad, simplemente que la forma en que la estamos midiendo permite detectar al paciente en un estado más avanzado de fragilidad cuando efectivamente está en riesgo de morir o de hospitalizarse.

Estos resultados, para el caso de una cirugía pueden ser adecuados en términos de pronóstico, sin embargo, no ha sido evaluada para el seguimiento tras una intervención de los pacientes que clasifica como frágiles.

Sabemos que fragilidad no es lo mismo que alteración funcional ni comorbilidad, aunque hay ocasiones en que pueden sobreponerse ${ }^{22}$, lo cual se corrobora en nuestros resultados, tal como muestra el modelo que evalúa mortalidad ajustado por patología médica, la fragilidad medida por nuestra escala se asocia significativamente con este desenlace en forma independiente.

Dentro de las limitaciones del estudio podemos mencionar que no se evaluó el posible deterioro funcional que podrían presentar los pacientes frágiles posterior a una cirugía, lo cual sería interesante analizar en estudios posteriores.

Actualmente estamos en proceso de evaluación de nuestra herramienta en distintas poblaciones y así poder dar más evidencia de validez externa.

En conclusión, la escala RAI-M es una herramienta corta y de fácil aplicación para detectar fragilidad en un contexto preoperatorio. Debe ser evaluado tanto su validez externa en otros centros, como la posibilidad de utilizarla en otros contextos clínicos. 


\section{Anexo 1}

\section{Índice de análisis de riesgo modificado (IAR-M)}

\section{Instrucciones: en primer lugar, deberá responder algunos datos personales}

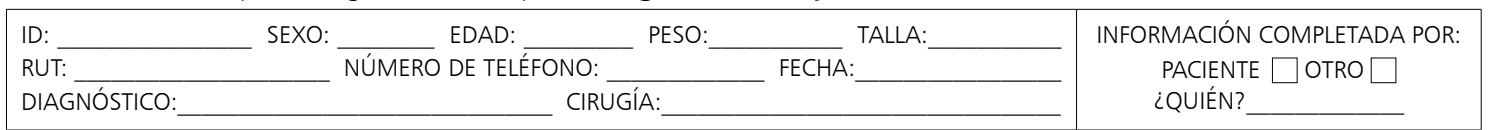

A continuación, deberá responder algunas preguntas sobre su historia médica y su vida diaria. Por favor, responda "Sí" o "No" a las siguientes preguntas.

\begin{tabular}{|c|c|}
\hline \multicolumn{2}{|l|}{ Historia Médica } \\
\hline A usted lo han diagnosticado de: & (todas sí: 1 no: 0 ) \\
\hline Hipertensión & $\square$ sí $\square$ No \\
\hline Diabetes & $\square$ Sí $\square$ No \\
\hline Cáncer (excluir cánceres menores como cáncer de piel) & $\square$ Sí $\square$ No \\
\hline EPOC & $\square$ Sí $\square$ No \\
\hline IAM & $\square$ Sí $\square$ No \\
\hline Insuficiencia cardíaca & $\square$ Sí $\square$ No \\
\hline Angina & $\square$ Sí $\square$ No \\
\hline Asma & $\square$ Sí $\square$ No \\
\hline Artritis & $\square$ sí $\square$ No \\
\hline ACV & $\square$ sí $\square$ No \\
\hline ERC & $\square$ Sí $\square$ No \\
\hline \multicolumn{2}{|l|}{ Componente físico y nutricional } \\
\hline 1. En los últimos 6 meses, ¿ha bajado 3 kilos o más sin hacer dieta? & $\square$ sí $\square$ No \\
\hline 2. En los últimos 6 meses ¿ha notado que la ropa le queda más suelta? & $\square$ sí $\square$ No \\
\hline 3. ¿Usted cree o le han dicho que está comiendo menos? & $\square$ Sí $\square$ No \\
\hline 4. ¿Usted se siente cansada la mayor parte del tiempo? & $\square$ Sí $\square$ No \\
\hline 5. ¿Usted puede subir un piso de escalera (10 escalones) sin descansar? & $\square$ Sí $\square$ No \\
\hline 6. ¿Usted puede subir un piso de escalera (10 escalones) sin ayuda? & $\square$ Sí $\square$ No \\
\hline \multicolumn{2}{|l|}{ Componente cognitivo } \\
\hline 7. ¿A usted le han dicho que tiene problemas de memoria? & $\square$ sí $\square$ No \\
\hline 8. ¿Usted siente que tiene menos memoria que otras personas de su misma edad? & $\square$ sí $\square$ No \\
\hline 9. ¿A usted se le olvidan compromisos? (ej. citas al doctor) & $\square$ sí $\square$ No \\
\hline 10. ¿A usted le preocupa su problema de memoria como para consultar por ello? & $\square$ Sí $\square$ No \\
\hline \multicolumn{2}{|l|}{ Componente afectivo } \\
\hline 11. ¿Usted ha sentido poco interés por hacer sus cosas? & $\square$ sí $\square$ No \\
\hline 12. ¿Usted se ha sentido triste o deprimido frecuentemente? & $\square$ sí $\square$ No \\
\hline $\begin{array}{l}\text { 13. En los últimos } 6 \text { meses, ¿usted cree que ha dejado de hacer las cosas que antes disfrutaba? (ej. tejer, jugar } \\
\text { cartas, hablar con sus amigos o vecinos) }\end{array}$ & $\square$ sí $\square$ No \\
\hline 14. ¿Usted se siente solo? & $\square$ sí $\square$ No \\
\hline \multicolumn{2}{|l|}{ Componente funcional } \\
\hline 15. Si usted está solo en su casa, ¿sería capaz de ducharse? (ej. entrar a la ducha, jabonarse) & Sí: 0 No: 1 \\
\hline 16. ¿Usted es capaz de vestirse solo? (ej. elegir su ropa, poner y sacar la ropa) & Sí: 0 No: 1 \\
\hline 17. ¿Usted usa ayuda para caminar? (ej. usa bastón o burrito) & Sí: 1 No: 0 \\
\hline 18. ¿Usted es capaz de caminar de su pieza al baño sin ayuda de otros? & Sí: 0 No: 1 \\
\hline 19. ¿Usted puede tomar sus medicamentos por sí solo? (ej. tomar la dosis adecuada en el horario indicado) & Sí: 0 No: 1 \\
\hline 20. ¿Es capaz de manejar su dinero por sí solo? (ej. pagar las cuentas, hacer un cheque) & Sí: 0 No: 1 \\
\hline 21. ¿Usted ha presentado caídas al desplazarse en su rutina diaria los últimos 6 meses? & Sí: 1 No: 0 \\
\hline \multicolumn{2}{|l|}{ Componente social } \\
\hline 22. ¿Usted tiene a alguien que lo pueda cuidar en los 7 días posteriores a su cirugía? & Sí: 0 No: 1 \\
\hline 23. ¿Usted tiene a alguien que lo ayude en caso de tener algún problema? & Sí: 0 No: 1 \\
\hline 24. ¿Usted tiene pensión o dinero para comprar sus fármacos sin problemas? & Sí: 0 No: 1 \\
\hline 25. ¿Usted tiene quien lo acompañe a sus controles postoperatorios? & Sí: 0 No: 1 \\
\hline
\end{tabular}


Anexo 2

Escala de Edmonton (EFS)

\begin{tabular}{|c|c|c|c|c|}
\hline Dominio de fragilidad & Ítem & 0 punto & 1 punto & 2 puntos \\
\hline Cognitivo & $\begin{array}{l}\text { Por favor imagine que este círculo pre- } \\
\text { dibujado es un reloj. Me gustaría que } \\
\text { pusiera los números en las posiciones } \\
\text { correctas y luego poner las manillas (o } \\
\text { manecillas) para indicar la hora "Las } \\
\text { once con diez minutos" }\end{array}$ & Sin errores & $\begin{array}{l}\text { Errores } \\
\text { mínimos de } \\
\text { espaciado }\end{array}$ & Otros errores \\
\hline \multirow[t]{2}{*}{ Estado de Salud General } & $\begin{array}{l}\text { En el último año, ¿cuántas veces ha } \\
\text { estado hospitalizado? }\end{array}$ & 0 & $1-2$ & $\geq 3$ \\
\hline & En general, ¿cómo describiría su salud? & Excelente & Razonable & Mala \\
\hline Independencia funcional & $\begin{array}{l}\text { ¿Con cuántas de las siguientes activida- } \\
\text { des necesita ayuda? (preparar la comi- } \\
\text { da, compras, transporte, comunicación } \\
\text { telefónica, cuidado del hogar, lavado } \\
\text { de ropa, manejo de dinero, tomar me- } \\
\text { dicamentos) }\end{array}$ & $0-1$ & $2-4$ & $\geq 4$ \\
\hline Soporte social & $\begin{array}{l}\text { ¿Cuando usted necesita ayuda, puede } \\
\text { contar con alguien que esté dispuesto y } \\
\text { disponible para atender sus necesidades } \\
\text { o problemas? }\end{array}$ & Siempre & A veces & Nunca \\
\hline \multirow[t]{2}{*}{ Uso de medicamentos } & $\begin{array}{l}\text { ¿Usa } 5 \text { o más medicamentos en el día } \\
\text { a día? }\end{array}$ & No & Sí & \\
\hline & $\begin{array}{l}\text { En ocasiones, ¿se le olvida tomarse los } \\
\text { medicamentos? }\end{array}$ & No & Sí & \\
\hline Nutrición & $\begin{array}{l}\text { Recientemente, ¿Ha perdido peso como } \\
\text { para que su ropa le quede suelta? }\end{array}$ & No & Sí & \\
\hline Ánimo & $\begin{array}{l}\text { ¿Se siente con frecuencia triste o de- } \\
\text { primido? }\end{array}$ & No & Sí & \\
\hline Continencia & $\begin{array}{l}\text { ¿Tiene algún problema con el control } \\
\text { para orinar, es decir puede contener la } \\
\text { orina si así lo desea? }\end{array}$ & No & Sí & \\
\hline Rendimiento funcional & $\begin{array}{l}\text { Me gustaría que se sentara en esta } \\
\text { silla con su espalda y brazos relajados. } \\
\text { Luego, cuando yo diga "YA", por favor } \\
\text { párese y camina a un ritmo cómodo } \\
\text { y seguro a la marca del suelo (aproxi- } \\
\text { madamente a } 3 \text { metros de distancia), } \\
\text { regrese a su silla y se sienta }\end{array}$ & $\begin{array}{l}0-10 \\
\text { segundos }\end{array}$ & $\begin{array}{l}11-20 \\
\text { segundos }\end{array}$ & $\begin{array}{c}\text { Uno de: }>20 \\
\text { s, paciente } \\
\text { no quiere } \\
\text { o requiere } \\
\text { asistencia }\end{array}$ \\
\hline Totales & $\begin{array}{l}\text { El puntaje final es la suma de las colum- } \\
\text { nas totales }\end{array}$ & & & \\
\hline
\end{tabular}


Anexo 3

\section{Índice de fragilidad de Rockwood}

\author{
Sindromas geriátricos \\ 1. Incontinencia \\ 2. Polifarmacia ( 5 o más medicamentos al día) \\ 3. Baja de peso en últimos 6 meses \\ 4. Caída en los últimos 6 meses

\section{Morbilidad} \\ 5. Hipertensión arterial \\ 6. Diabetes mellitus \\ 7. Enfermedad pulmonar crónica \\ 8. Insuficiencia cardiaca \\ 9. Antecedente de accidente cerebrovascular \\ 10. Artritis \\ 11. Enfermedad renal crónica \\ 12. Antecedente de cáncer \\ 13. Antecedente de angina o IAM

\section{Evaluación físico y nutricional} \\ 14. IMC (normal o alterado) \\ 15. Dinamometría ambas manos (límites descritos para \\ hombres y mujeres) \\ 16. Circunferencia de pantorrilla (31 cm como límite) \\ 17. Test up and Go (10 segundos como límite) \\ 18. Sensación de cansancio \\ 19. Ropa más suelta
}

\section{Evaluación cognitivo}

20. Test del reloj

21. Presenta problemas de memoria autoreferido

22. Presenta problemas de memoria referido por otros

\section{Funcionalidad}

23. Ducharse sin ayuda

24. Vestirse sin ayuda

25. Ayuda para caminar

26. Toma medicamentos por si solo

27. Maneja dinero por si solo

28. Puede subir un piso de escalera sin ayuda

\section{Evaluación área afectivo y social}

29. Sentimiento de tristeza o depresión

30. Percepción de salud

31. Soporte social
Anexo 4

\section{Descripción de mediciones objetivas}

\section{Fuerza prensora de la mano por dinamometría}

Previo a la cirugía se midió la fuerza prensora en ambas manos con dinamómetro Jamar, validado a nivel internacional como el estándar de oro.

Se utilizaron las recomendaciones estándar.

El dinamómetro Jamar fue utilizado en la segunda posición.

El paciente en posición sentado, con flexión del codo en $90^{\circ}$, apoyo del antebrazo y con la muñeca entre 0 a $30^{\circ}$ de dorsiflexión, se le pedía al paciente que realizaran el máximo esfuerzo prensor en un solo tiempo.

El investigador realizaba estímulo motivacional mientras se realizaba la acción.

Se tomaron dos mediciones por cada mano, con descanso de dos minutos entre cada medición y se utilizó el valor más alto para el análisis.

\section{Medición de Timed Up and Go}

Se disponía de una silla, con un punto a 3 metros de distancia para realizar la medición.

Se le daba la instrucción al paciente de levantarse de la silla sin apoyo de los brazos, caminar lo más rápido posible, sin correr y con seguridad en la marcha, llegando hasta el punto de 3 metros de distancia, y luego dar la vuelta y regresar a sentarse en la misma silla, sin utilizar los brazos.

Esta acción era cronometrada por el investigador.

\section{Medición de perímetro de pantorrilla}

En posición sentada, se medía el contorno de la pantorrilla de ambas piernas, con una huincha de medir.

La medición más alta es la considerada para el análisis.

\section{Referencias}

1. Instituto Nacional de Estadística. Estimaciones y proyecciones de la población de Chile 1992-2050 total país, síntesis de los resultados. 2018. Disponible en http:// www.ine.cl/estadisticas/demograficas-y-vitales [Consultado el 19 de julio de 2019].

2. Fried L, Tangen C, Walston J, Newman A, Hirsch C, Gottdiener J, et al. Frailty in older adults: evidence for a

phenotype. J Gerontol A Biol Sci Med Sci 2001; 56A (3): M146-56.

3. Robinson T, Walston J, Brummel N, Deiner S, Brown Iv C, Kennedy M, et al. Frailty for surgeons: review of a National Institute on Aging Conference on Frailty for specialists. J Am Coll Surg 2015; 221 (6): 1083-92.

4. Rockwood K, Mitnitski A. Frailty in relation to the accumulation of deficit. J Gerontol A Biol Sci Med Sci 2007; 62A (7): 722-7. 
5. Rolfson D, Majumdar S, Tsuyuki R, Tahir A, Rockwood K. Validity and reliability of the Edmonton Frail Scale. Age Ageing 2006; 35: 526-9.

6. Cohelo S, Veiga F, Ramos T, Haas V, Spadoti R, Partezani R. Adaptación cultural y validez de la Edmonton Frail Scale-EFS en una muestra de ancianos brasileños. Rev Lat Am Enfermagem 2009; 17: 1-8.

7. Ramírez J, Cadena M, Enrique M. Aplicación de la escala de fragilidad de Edmonton en población colombiana: comparación con los criterios de Fried. Rev Esp Geriatr Gerontol 2017; 52 (6): 322-5.

8. Rockwood K, Andrew M, Mitnitski A. A comparison of two approaches to measuring frailty in elderly people. J Gerontol A Biol Sci Med Sci 2007; 62A (7): 738-43.

9. Mitnitski A, Mogilner A, Rockwood K. Accumulation of deficit as a proxy measure of aging. The Scientific World 2001; 1: 323-36.

10. Searle S, Mitnitski A, Gahbauer E, Gill T, Rockwood K. A Standard procedure for creating a frailty index. GMC Geriatr 2008; 8: 24.

11. Syddall H, Cooper C, Martin F, Briggs R, Aihie Sayer A. Is grip strength a useful single marker of frailty? Age Ageing 2003; 32 (6): 650-6.

12. Documento de Organización Panamericana de la Salud: Encuesta sobre salud, bienestar y envejecimiento en Santiago, Chile. Albala C, García C, Lera L, 2000, Disponible en http://iris.paho.org/xmlui/bitstream/ handle/123456789/741/9275316090.pdf? sequence $=1$ [Consultado el 31 de julio de 2019].

13. Landi F, Onder G, Russo A, Liperoti R, Tosato M, Martone $\mathrm{A}$, et al. Calf circumference, frailty and physical performance among older adults living in the community. Clin Nutr ESPEN 2014; 33 (3): 539-44.

14. Cesari M, Leeuwenburgh C, Lauretani F, Onder G, Ban- dinelli S, Maraldi C, et al. Frailty syndrome and skeletal muscle: results from the invecchiare in Chianti Study. Am J Clin Nutr 2006; 83: 1142e8.

15. Podsiadlo D, Richardson S. The Timed "Up \& Go": a Test of basic functional mobility for frail elderly persons. J Am Geriatr Soc 1991; 39 (2): 142-8.

16. Porock D, Parker-Oliver D, Zweig S, Rantz M, Mehr D, Madsen R, et al. Predicting death in the nursing home: development and validation of the 6-month minimum data set mortality risk index. J Gerontol A Biol Sci Med Sci 2005; 60 (4): 491-8.

17. Hall D, Arya S, Schmid K, Blaser C, Carlson M, Bailey $\mathrm{T}$, et al. Development and initial validation of the risk analysis index for measuring frailty in surgical populations. JAMA Surg 2016; 15213: 1-8.

18. Hall D, Arya S, Schmid K, Carlson M, Lavedan P, Bailey $\mathrm{T}$, et al. Association of a frailty screening initiative with postoperative survival at 30,180 , and 365 days. JAMA Surg 2016; 378 (9800): 1408-13.

19. Nunnally J. Psychometric Theory (2nd ed). New York: McGraw-Hill Edition; 1978.

20. Thorndike R. Applied Psychometrics. Boston: Houghton-Mifflin Edition; 1982.

21. Guilford J. Psychometric methods (2nd ed.). New York: McGraw-Hill Edition; 1954.

22. Kline P. Psychometrics and psychology. London: Acaderric Press Edition; 1979.

23. Robertson D, Savva G, Kenny R. Frailty and cognitive impairment: a review of the evidence and causal mechanisms. Ageing Res Rev 2013: 12 (4): 840-51.

24. Fried L, Tangen C, Walston J, Newman A, Hirsch C, Gottdiener J. Frailty in older adults: evidence for a phenotype. J Gerontol A Biol Sci Med Sci 2001; 56 (3): M146-56. 